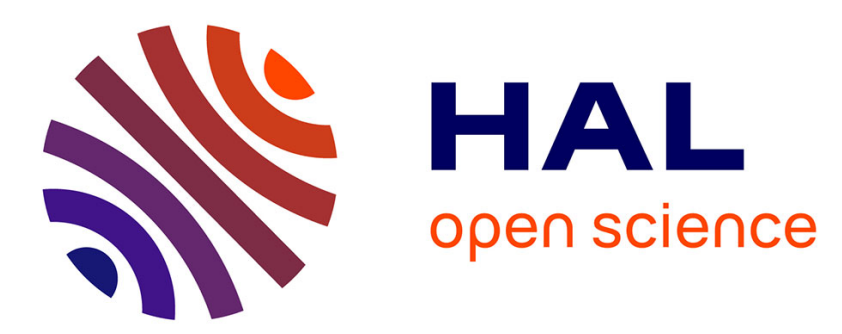

\title{
The (homo)morphism concept: didactic transposition, meta-discourse and thematisation
}

Thomas Hausberger

\section{To cite this version:}

Thomas Hausberger. The (homo)morphism concept: didactic transposition, meta-discourse and thematisation. International Journal of Research in Undergraduate Mathematics Education, 2017, 3 (3), pp.417-443. 10.1007/s40753-017-0052-7 . hal-01408419

\section{HAL Id: hal-01408419 \\ https://hal.science/hal-01408419}

Submitted on 20 Feb 2021

HAL is a multi-disciplinary open access archive for the deposit and dissemination of scientific research documents, whether they are published or not. The documents may come from teaching and research institutions in France or abroad, or from public or private research centers.
L'archive ouverte pluridisciplinaire HAL, est destinée au dépôt et à la diffusion de documents scientifiques de niveau recherche, publiés ou non, émanant des établissements d'enseignement et de recherche français ou étrangers, des laboratoires publics ou privés. 


\title{
The (homo)morphism concept: didactic transposition, meta-discourse and thematisation
}

\author{
Thomas Hausberger
}

Received: date / Accepted: date

\begin{abstract}
This article focuses on the didactic transposition of the homomorphism concept and on the elaboration and evaluation of an activity dedicated to the teaching of this fundamental concept in Abstract Algebra. It does not restrict to Group Theory but on the contrary raises the issue of the teaching and learning of algebraic structuralism, thus bridging Group and Ring Theories and highlighting the phenomenon of thematisation. Emphasis is made on epistemological analysis and its interaction with didactics. The rationale of the isomorphism and homomorphism concepts is discussed, in particular through a textbook analysis focusing on the meta-discourse that mathematicians offer to illuminate the concepts. A piece of didactic engineering, informed by the preceding analysis and using epistemological insight as a meta-lever, is presented. The empirical results of a classroom realisation are discussed in the epistemological framework, through comparison of the a priori and a posteriori analysis. This experiment shows both the potential and the difficulties in connecting the homomorphism formalism to cognitive processes of comparison and identification.
\end{abstract}

Keywords Abstract Algebra · homomorphism concept · algebraic structuralism $\cdot$ didactic transposition $\cdot$ thematisation

\section{Introduction}

In what follows, the term "Abstract Algebra" is taken to mean the discipline devoted to the study of algebraic structures according to the new paradigm established after the publication of Waerden (1930). Thus Abstract Algebra (Groups, Rings and Fields) is taught at Montpellier University (and in France in general) at third-year university level. The difficulties faced by students

T. Hausberger

Université de Montpellier CC 051 - Place Eugène Bataillon F-34095 Montpellier Cedex 5

E-mail: thomas.hausberger@umontpellier.fr 
of Abstract Algebra are acknowledged by several authors (Leron \& Dubinsky, 1995; Nardi, 2000; Durand-Guerrier, Hausberger, \& Spitalas, 2015) and reflect a "transition problem" (Gueudet, 2008) which occurs inside the university curriculum. Transitions from "concrete to abstract", in the context of analysis, have been studied by Winsløw (2008) using the Anthropological Theory of Didactics as a theoretical framework. A similar approach is under investigation for Abstract Algebra, based on the notion of "structuralist praxeology" that I have introduced (Hausberger, 2016b).

The approach that I am presenting in this paper is based on an epistemological analysis of algebraic structuralism (Hausberger, 2012) and the investigation of students' difficulties which are inherent to structuralist thinking. The epistemological analysis can also serve as an anchor point for the development of teaching strategies, in particular to introduce "meta" aspects in the teaching of Abstract Algebra. In this approach, students are helped to make sense of structuralist concepts, thanks to epistemological clarifications as a meta discourse and to reflexive thinking. This is justified by the identification, from an epistemological point of view, of the FUGS (formalising, unifying, generalising \& simplifying) character (Robert, 1987) of Abstract Algebra concepts. Obstacles regarding the built-up of teaching strategies, using traditional didactic tools such as the "theory of didactical situations" (Brousseau, 1986) or the "tool-object dialectic" (Douady, 1986), can be analysed as consequences of their epistemological nature as FUGS (Rogalski, 1995). Such a "meta lever" has been used previously by Dorier, Robert, Robinet, and Rogalski (2000) in the case of Linear Algebra.

In (Hausberger, 2012), I underlined the fact that several levels of unification have to be distinguished in the context of algebraic structures: at level 1 , a general theory applies to quite different objects sharing a common feature (for instance Group Theory), at level 2, the axiomatic presentation of structures is conducted in a uniform way (leading to structural questions and methods) and puts forward bridges between structures, at level 3, what has been previously a form (structures) is fully taken and studied as object in a superior level of organisation (this is Category Theory or any other mathematical meta-theory ${ }^{1}$ of structures). I made the assumption that the introduction of meta activities of level 2 would facilitate the access to modern structuralist expositions of algebraic structures. In the case of Linear Algebra, Dorier introduced only meta discourse of level 1 , which is justified by the fact that a single structure is involved and that it is the first abstract structure that students encounter and theorise at university.

This article focuses on the (homo)morphism and isomorphism concepts which are central in Abstract Algebra. I will follow the methodology of didactical engineering (Artigue, 2009) for research-based action on educational systems, being both the teacher and the researcher in this study. I will thus begin with an epistemological analyses of these concepts: I will point out that

\footnotetext{
${ }^{1}$ the word "meta" being used here in a different context and with a slightly different meaning. Nevertheless, meta-theories and meta-activities meet in so far as they both introduce a reflexive point of view
} 
the rewriting of classical algebra in terms of structures has been operated by mathematicians in an act of abstraction that philosophers Cavaillès (2008) and Lautmann (2006) called "thematisation" and which is the key to level 2 unification. This phenomenon inspired Piaget's "reflective abstraction" (Piaget, 1972), which in turn fostered further developments in Mathematics Education (Dubinsky, 1991) of a cognitive nature. By contrast, my epistemological approach aims at understanding how thematisation is achieved as a particular organisation of knowledge around the central concept of homomorphism. I will then present an analysis of selected textbooks in order to study the phenomenon of thematisation at the level of the didactic transposition. This institutional analysis will complement the epistemological analyses in order to inform the next step of task design. In particular, I will focus on the metadiscourse that mathematicians use in order to point out the rationale of the homomorphism and isomorphism concepts. Finally, I will present the piece of didactical engineering that has been produced, in this case a situation dedicated to the learning of ring-homomorphisms, using the meta lever, to students who have already encountered group-homomorphisms, and by discussing the empirical results obtained after its classroom realisation. Compared to previous studies (Leron et al., 1995; Nardi, 2000) which restrict attention to homomorphisms in Group Theory, the extension to Ring Theory allows a didactical analysis of thematisation as a cross-structure process.

\section{Idealisation and thematisation}

According to Cavaillès (2008), idealisation and thematisation are two fundamental processes of abstraction that are involved in structuralist thinking. As abstraction has two faces, a mathematical-logical face and a psychologicalcognitive face, I will elaborate on both aspects. Algebraic structures are concepts which I will see as historical products of the mind's activity.

Following Cavailles, Sinaceur (2014, p. 94-95) describes idealisation as a process which amounts to:

leaving aside or discarding all other aspects, especially specific substantial or space-time aspects. [...] it comes down to extracting a form from sundry situations [...] idealization follows from seeing or guessing some invariant basic properties attached to a plurality of apparently heterogeneous situations and it leads to a unifying view of the different domains on which we perform the same type of operations;

whereas thematisation is about:

isolating some property or some set of properties of the operation(s) under consideration and viewing them on their own, i.e., transforming the selected conjunction of predicates into a thought-object [...] Peirce called this kind of transformation "reflective" or "hypostatic" abstraction, Husserl called it "thematisation" [...] Thematisation is essential from passing [...] from the study of a structure $\Sigma$ on the set $S$ to the 
study of the structure $\Sigma$ in its own right, i.e., to the study of a class of homomorphisms between structures of the type $\Sigma$. [...] Attention is paid to the homomorphisms rather than to the sets that are respectively source and target of them.

Cavailles' account of algebraic structuralism, as a philosophical dialectic between form and content, much inspired Piaget (1972, p. 70) who considered that "the whole of mathematics may therefore be thought of in terms of the construction of structures" according to reflective abstraction which he saw as an encapsulation of forms as new contents. In other words, "actions or operations become thematised objects of thought or assimilation" (Piaget, 1985, p. 49). From this trend follows the encapsulation of a process into an object as described by Dubinsky (1991) in APOS theory.

More developments may be seen in the Mathematics Education literature: for instance, structural abstraction (Scheiner \& Pinto, 2014) considered as a "movement from particular to unity in terms of complementarizing particularized meaningful components/structure into a whole". The idea of structure as an integrated whole of interrelated elements, which originated from algebraic practises, has become a standard model used to apprehend phenomena in human sciences in general, with the popularisation of structuralist views. It is certainly fruitful and pertinent in order to produce cognitive analyses of mathematical activity, but the point I wish to make is that, in order to analyse and understand the mathematical phenomena that inspired Piaget, epistemological analyses should be carried further: reflective abstraction and thematisation (the word popularised by Cavailles among French philosophers) are not entirely synonymous; the encapsulation does not describe the mathematical organisation of the thematised objects of thought according to a "theme", a methodological paradigm.

Simpson and Stehlíková (2006) have explored the "acquisition of structural sense" (p. 348) in relation to "coming to understand a commutative ring". They pointed out several "shifts of attention" required for the transition from objects to abstract structures and focused especially on "attending to the interrelationships between elements in the set which are consequences of the operation" (loc. cit. p. 352). This is the relational point of view (emphasis on relations rather than elements) which led to the formal axiomatic point of view popularised by Hilbert, as abstraction is often closely tied up with axiomatisation and symbolisation in modern mathematical practises. This consideration certainly explains the other shifts of attention that Simpson et al. have highlighted.

Nevertheless, another shift of attention seems neglected, the shift due to Noether which "changed the face of algebra" (E. Artin, 1962, p. 555) and brought to the fore the notion of homomorphism, as described by Sinaceur. The next section will therefore be dedicated to an in-depth analysis of the isomorphism and homomorphism concepts with a view to a better understanding of the phenomenon of thematisation in the mathematical practises within Abstract Algebra. 
3 Epistemological insight on the homomorphism and isomorphism concepts

\subsection{Structure-preserving functions}

Abstract Algebra teachers, after giving the formal definition of homomorphism and isomorphism, often mention that these are structure-preserving functions. Such statements are part of the epistemological arguments that are provided to help students understand the rationale of the concepts. On the other hand, teachers never mathematically define any concept of structure. Let us explain this a priori abnormal phenomenon by giving a few details concerning the didactic transposition (Chevallard, 1985) of the notion of structure.

In his attempt to give a historical account, Corry (2004) makes the distinction between "body of knowledge" and "image of knowledge". The notion of structure takes its origin in the latter:

This textbook [Moderne Algebra] put forward a new image of the discipline that implied in itself a striking innovation: the structural image of algebra. In the forthcoming account, it is this specific, historically conditioned image of mathematical knowledge that will be considered as implicitly defining the idea of a mathematical structure (Corry, 2004, p. 8).

Van der Waerden did not give any comment, formal or non-formal, on what he meant by a "structure". Bourbaki, on the contrary, gave a formal-axiomatic elucidation of the concept of mathematical structure in the first book of his treatise "Elements de mathématiques" dedicated to set theory and published in the 1950s. In parallel, he promoted (Bourbaki, 1948, reed. 1997) the structural image of mathematics within the noosphere (Chevallard, 1985). Yet, Bourbaki's definition hardly played any role in the exposition: it only provided a general framework which in fact did not prove to be mathematically functional (Mac Lane, 1996; Corry, 2004, p. 324), unlike Category Theory which is very advanced and too elevated a viewpoint for the present purpose. Therefore no definition at all is given in more recent textbooks.

As a consequence, students are supposed to learn by themselves and by the examples what is meant by a structure. This may require a considerable length of time in order to acquire a "structural sense" (Simpson \& Stehlíková, 2006). In this context, how may a sentence like "a homomorphism is a structurepreserving function" be interpreted? On the basis of formal definitions of vector spaces or groups, the students may understand that structures refer to sets of axioms. This reflects the fact that the notion of structure is an outgrowth of the widespread use of the axiomatic method ${ }^{2}$. As algebraic systems of axioms involve operations such as + , the homomorphic condition being an

\footnotetext{
${ }^{2}$ Historically, this method was initially deployed by Hilbert to remedy the imperfections of Euclid's axioms for geometry and give rigorous descriptions of mathematical objects suitable for applying the demonstrative apparatus. In this trend, logical use of the method is about questioning consistency, mutual independence, completeness of the set of axioms, in order to give foundations to the theory. In the context of Abstract Algebra, we are concerned with a
} 
equality $f(x+y)=f(x)+f(y)$, structure-preserving becomes synonymous with operation-preserving. We will see in the sequel that this contrasts with the understanding of a homomorphism as a relation-preserving map. Therefore, it is doubtful that students would be able to give a formal definition of homomorphism in a non-algebraic context, for instance a homomorphism of ordered sets.

Moreover, the word "structure" is used in fact in different contexts with a different meaning, which may induce some confusion: mathematicians describe the different isomorphism classes for a given structure and say for instance that the abstract group-structure of $\mathbb{Z} / 3 \mathbb{Z}$ is that of a cyclic group of order 3. Finally, mathematicians call "structure-theorem" a result describing the way an object can be reconstructed from simpler objects of the same type. Altogether, this investigation of the meta-concept of structure certainly contributes to break the illusion of transparency (Artigue, 1991) concerning the concept of homomorphism as a structure-preserving function and the idea of a mathematical structure in general.

\subsection{Group isomorphisms and the abstract group concept}

According to historians (Wussing, 2007), the recognition of the central role played by the group concept in permutation theory and in the unification of the different geometries allowed the development of the group structure as the first abstract mathematical structure, before the beginning of the 20th century. The unification of the different historical sources of groups was operated by Dyck (1882) who introduced the abstract group concept from "generating operations" and the consideration of their "mutual relations".

The abstract group concept is closely related to the concept of groupisomorphism: disregarding the particular nature of elements (this is idealisation), which is made possible through the consideration of relations between elements and the use of symbolic algebra, amounts to considering the different instances (models) of groups up to isomorphism. The isomorphism concept is an equivalence relation, which allows the consideration of classes of isomorphic groups which are seen as equivalent. A classification is a description of the corresponding partition: for instance, Group Theory will classify groups of a given order. This reflects the fact that idealisation is often closely tied to the logical technique of subsumption, formalised by Russell (1903) under the name "principle of abstraction". Indeed, this principle is the key to conceptformation. Nevertheless, mathematical abstraction has many facets and cannot be reduced to logical abstraction (Sinaceur, 2014).

Van Dyck's description of a class is not based on a given representative but in the consideration of generators and relations. The modern rigorous formalisation of this idea requires the notion of free group. Just as any group is the

more immanent use of axiomatics: properties of concrete objects are abstracted in order to acquire a better understanding of ideas underlying mathematical constructions and proofs. This allows a unification of objects and methods, leading to the structural point of view. 
quotient of the free group on the generators by the smallest normal subgroup containing the relations, making a quotient is equivalent to introducing more relations. Therefore the idea of relation is useful to understand quotients: this should help the students to interpret, for instance in the ring setting, the quotient $\mathbb{Z}[X] /(10 X-1)$ as a ring isomorphic to the decimal fractions. This is another argument for reestablishing the importance of the notion of relations which tend to be hidden behind the notion of operation.

\subsection{Noether's set-theoretic foundation of Algebra}

The concept of isomorphism as an equivalence relation needs to be distinguished from that of a function (with properties). I will now focus on the latter, which relies on the notion of homomorphism and is the heritage of Noether who developed

[...] what she called her set-theoretic foundations for algebra. This was not what we now call set theory. [...] Rather, her project was to get Abstract Algebra away from thinking about operations on elements, such as addition or multiplication of elements in groups or rings. Her algebra would describe structures in terms of selected subsets (such as normal subgroups of groups) and homomorphisms (McLarty, 2006, p. 188).

One can make the hypothesis that Noether's new conceptual approach is a major epistemological difficulty in the learning of Abstract Algebra: the epistemological transition from thinking about operations on elements to thinking in terms of selected subsets and homomorphisms may be seen as an explanatory factor of the transition problem at the third year University level. It is a new "shift of attention" that I see, following (Sinaceur, 2014), as closely related to thematisation. The difference between von Dyck and Noether is therefore related to the distinction between idealisation and thematisation.

Dedekind's theory of ideals, in which the divisibility relation between (algebraic) integers was replaced by inclusion of ideals, certainly contributed to the transition from an arithmetical conception of algebra to the set-theoretic conception. But Noether's chief tools were isomorphism theorems and she made it obvious that this applied for different kinds of structures. As an illustration of the generality of the principle:

Ideals bear the same relation to ring homomorphisms as do normal subgroups to group homomorphisms. Let us start from the notion of homomorphism (Waerden, 1930, 2nd ed. 1949, p. 51).

Compared to von Dick's approach which restricts to level 1 unification (referring to the introduction), Noether's point of view is the key to level 2 structuralism: this indeed allows a unified treatment of structures. It proved in history to be a major breakthrough leading to a complete rewriting of algebra (in terms of newly-forged concepts that emerged from the new methodology: noetherian rings, principle-ideal domains, etc.). 
Noether considered only surjective homomorphisms, denoted $M \sim \bar{M}$ (McLarty, 2006, p. 205; the functional notation $f: M \rightarrow \bar{M}$ comes from topology), and correlated them to distinguished classes of subsets through the First Isomorphism Theorem, which is stated nowadays as follows (Nardi, 2000, p. 179):

Theorem 1 (First Isomorphism Theorem for groups) Let $G, G^{\prime}$ be groups and $\phi: G \rightarrow G^{\prime}$ a homomorphism. If $K=\operatorname{ker} \phi$ then $G / K \sim \Im m \phi$. [The homomorphism is constructed by setting $\psi(K g)=\phi(g)$ ]

Historically, the rationale of the homomorphism concept is therefore threefold: (i) compared to the classical notion of isomorphism, it relates two objects $M$ and $\bar{M}$, the latter being isomorphic to a quotient of the former; (ii) this is a general procedure that applies to any structure and allows to relate, for instance, Group Theory and Ring Theory; (iii) the distinguished sets are the kernels of homomorphisms.

\subsection{Homomorphisms or morphisms?}

Are the two terms, homomorphism and morphism, synonymous? More types of morphisms are encountered by students: homeomorphisms, diffeomorphisms, monomorphisms, epimorphisms, endomorphisms, automorphisms, which may contribute to confusion ${ }^{3}$.

The morphism concept is a generalisation of the homomorphism concept beyond that of a structure-preserving function (which does not apply to morphisms in topology). This generalisation appeared with the development of Category Theory, by thematisation of concepts (such as morphism) idealised from the consideration of different mathematical structures. In a category, one is free to decide which maps are morphisms, these define the category together with a given type of objects. If morphisms tend to replace homomorphisms, beyond the pragmatic argument (morphism is a shorter term), it might be that Abstract Algebra practitioners are implicitly assuming that they are working in a specific algebraic category. A morphism of groups is a structure-preserving map but morphisms are not in general whereas homomorphisms always are. This explains the pertinence of the terminology "homomorphism" for a thematisation of this concept in the algebraic setting and the didactical importance of discussing the etymology of the mathematical vocabulary with students.

\footnotetext{
3 as is visible, for instance, on the mathematical forum http://www.les-mathematiques .net
} 
4 The didactic transposition of the isomorphism and homomorphism concepts: analysis of selected textbooks

\subsection{Methodology}

The aim of this section is to view a few standard textbooks in Abstract Algebra under the light of the preceding epistemological analysis, focusing on the phenomenon of thematisation and the epistemological arguments that mathematicians put forward in order to discuss the rationale of the homomorphism and isomorphism concepts. This exploratory work will both shed light on the complex process of didactic transposition (Chevallard, 1985) of the homomorphism and isomorphism concepts, and bring bricks for the engineering of an activity dedicated to the discussion with students of ring-homomorphisms after a first course in Group Theory.

The chosen textbooks include van der Waerden's Modern Algebra (1930) as the first textbook available which takes its source in Noether's lectures and two standard textbooks: the textbook Algebra by M. Artin (1991) and the textbook "Algèbre T.1 : Groupes et Anneaux" 4 by Guin (1997). The selection is motivated by the presence of meta-discourse and the potential to bring contrasted results: my intent is not to draw general conclusions but again to break the illusion of transparency regarding algebraic concepts and the way mathematicians convey the "structural image" of Abstract Algebra. Michael Artin is the son of Emil Artin (to whom van der Waerden is also indebted as a source of his book) and a famous algebraic geometer, but he shows a philosophical proximity with the intuitionist Hermann Weyl. Guin is an expert in Category Theory and more of a formalist in the Bourbaki tradition.

The following aspects will be discussed:

- The "announced structuralist agenda": looking at the preface, does the author introduce a structural image of Abstract Algebra through a metadiscourse? Are structuralist goals set up in terms of FUGS concepts?

- The rationale of the isomorphism and homomorphism concepts : which comes first? Is a general notion given, building on the abstract concept of relation? How is it connected to normal subgroups and quotients? How are isomorphism theorems stated?

- Thematisation: How does the exposition of Ring Theory rely on the previous exposition of Group Theory? Is the formal analogy underlined in mathematical organisations and in the meta-discourse?

\subsection{The structuralist agenda}

Van der Waerden's Modern Algebra (Waerden, 1930) has been written as a treaty dedicated to the exposition of recent advances in algebra: "The chief purpose of this book is to introduce the reader into this whole world of con-

\footnotetext{
${ }^{4}$ Algebra 1: Group Theory and Ring Theory
} 
cepts. Within the scope of these modern ideas, the classical results and methods will find their due place." The first three chapters appear as a core whose goal consists in presenting the abstract point of view, with a focus on the two main structures of group and ring, substructures, isomorphism and homomorphism in connection with quotients, and whose unity is guaranteed by the formal analogy between the group and ring contexts. This kind of mathematical organisation emphasises what I called level 2 unification; it is emblematic of structuralist thinking and is the product of thematisation. Nevertheless, the arithmetic of abstract rings appears also as a strong motivation for the development of ideal theory.

On the contrary, M. Artin (1991) downplays the structuralist enthusiasm of the 1920s under the philosophical influence of Herman Weyl whom he cites to open the preface of his book: "Important though the general concepts and propositions may be with which the modern and industrious passion for axiomatizing and generelizing has presented us, in algebra perhaps more than everywhere else, nevertheless I am convinced that the special problems in all their complexity constitute the stock and the core of mathematics, and that to master their difficulties requires on the whole the hardest labor." As a consequence, in his note to the teacher, Artin suggests to "deemphasize the quotient group construction". Indeed, Artin is concerned about the ecology (Artaud, 1998) of this theoretical construct: "since the integers modulo $n$ form a ring, modular arithmetic isn't the ideal motivating example for quotient groups. The first serious use of quotient groups comes when generators and relations are discussed". Both philosophically and didactically, the introduction of such a conceptual apparatus cannot, according to Artin, rely on the idealisation and thematisation principles of abstraction most valued by structuralists. The main point should be its use to tackle concrete problems. According to Artin, the impossibility to implement a satisfying tool-object dialectic (Douady, 1986) in the teaching of quotient groups in elementary Group Theory, should, without the institutional habitus, lead to deferring the teaching of quotients to Ring Theory.

Guin motivates the abstract point of view in the preface using a few historical landmarks and pointing out the FUGS character of Abstract Algebra through a meta discourse around analogies and the economy brought by general statements. As we will see in the next section, his exposition differs from Artin's by the level of generality of mathematical statements and the problematisation of notions, which is more formal, building on Set Theory and having in mind Category Theory as the horizon. Much emphasis is made on the quotient structure. Therefore, although Abstract Algebra always presents an objects-structures dialectic, this dialectic manifests a tension which is visible in the didactic transposition that is operated by mathematicians. The domain of practise of the mathematician as well as his philosophical engagement impacts the transposition. 
4.3 The rationale of the isomorphism and homomorphism concepts

Van der Waerden defines the concept of isomorphism in general as a 1-1 correspondence preserving relations, and he denotes $\mathfrak{M} \cong \overline{\mathfrak{M}}$ homomorphic sets. Although a general notion of structure is not rigorously defined, the concept of relation appears as the anchor point for the thematisation of the isomorphism concept. From the point of view of semiotics, van der Waerden's notations (" $a b=c$ implies $\bar{a} \bar{b}=\bar{c}$ and vice versa") underline the conception of the isomorphism as a symmetric correspondence: this contrasts with the functional notation $\varphi(a b)=\varphi(a) \varphi(b)$ which emphasises the function rather than the relations between elements. The sign $\cong$ makes it clear that isomorphisms aim at comparing sets, and it points out a similarity. The automorphism concept is formally defined as an isomorphism between identical sets and is immediately connected to the intuitive notion of symmetry.

Similarly to van der Waerden, group isomorphisms precede homomorphisms in (M. Artin, 1991), but isomorphisms are operation-preserving correspondences rather than relation-preserving and no general notion of isomorphism is introduced. Great care is given to relate the concept of isomorphism as a correspondence to the functional asymmetrical point of view. With regard to semiotics, the notation $a^{\prime}$ replaces van der Waerden's $\bar{a}$ in order to save the bar for residue classes. Pragmatic reasons ("it is often convenient") are put forward in order to justify "informal identification" of isomorphic groups. The underlying principles of abstraction (idealisation and thematisation) are not discussed in further details, which is consistent with a general philosophy that does not present the structuralist horizon as a goal.

Contrary to van der Waerden and Artin, group homomorphisms are defined before group isomorphisms in Guin (1997), and Guin uses the term "morphism" preferably. The notion is introduced by the motivation that "in order to determine algebraic properties of a group, a very efficient strategy is to compare the group with another group we are already acquainted with". Even if $G$ is not a subgroup of $G^{\prime}$, both groups may be compared through "a map $f: G \rightarrow G^{\prime}$ so that we are brought back to the situation of a subgroup $f(G)$ in $G^{\prime}$. In order to do so, the map $f$ must be compatible with the group structure, that is with the law which defines the structure". As we can see, Guin is anchoring his meta-discourse on the structural image of algebra and the intuitive idea of comparing objects. Guin denotes the set of morphisms as $\operatorname{Hom}\left(G, G^{\prime}\right)$ and formally defines an isomorphism according to Category Theory as a map $f$ in $\operatorname{Hom}\left(G, G^{\prime}\right)$ which admits a reciprocal morphism, that is a map $g$ in $\operatorname{Hom}\left(G^{\prime}, G\right)$ such that $f \circ g=i d_{G^{\prime}}$ and $g \circ f=i d_{G}$. A proposition immediately connects this definition to the notion of bijective morphism. The symbol $\simeq$ which is used to denote an isomorphism is different from Artin's and van der Waerden's: the sign $\cong$ is saved for homeomorphisms in Topology.

The homomorphism concept is defined in (Waerden, 1930), again in full generality, as an onto map which preserves relations, and denoted $\mathfrak{M} \sim \overline{\mathfrak{M}}$ after Noether. This notation emphasises the connection between the homomorphism and isomorphism concepts, which is consistent with the statement 
that an isomorphism is a bi-unique mapping such that the property of homomorphism holds symmetrically. It should be noted that if this property holds in one direction, it holds both ways: van der Waerden does not give minimal conditions but chooses to underline symmetric aspects of objects and relations. By definition, a homomorphism is onto, which reflects the very purpose of homomorphisms: to establish isomorphic relations between $\overline{\mathfrak{M}}$ and quotients of $\mathfrak{M}$. Elements of $\overline{\mathfrak{M}}$ are now in 1-1 correspondence with classes of elements of $\mathfrak{M}$ and therefore "we shall find a very important one-to-one relation between homomorphisms and normal divisors". These considerations lead to the statement of the so-called "law of homomorphism for groups": "Any group $\overline{\mathfrak{G}}$ that is a homomorphic image of $\mathfrak{G}$ is isomorphic with a factor group $\mathfrak{G} / \mathfrak{e}$, where $\mathfrak{e}$ is the normal divisor of $\mathfrak{G}$ corresponding to the identity in $\overline{\mathfrak{G}}$. Conversely, $\mathfrak{G}$ is mapped homomorphically upon every factor group $\mathfrak{G} / \mathfrak{e}$, where $\mathfrak{e}$ is a normal divisor."

This result corresponds to the First Isomorphism Theorem (1st-IT) for Groups stated in the previous section of this paper. Nevertheless, a few distinctions should be pointed out: the main ostensives ${ }^{5}$ (Chevallard \& Bosch, 1999) in the 1st-IT are the kernel and the image of the homomorphism, so that the 1st-IT may be interpreted as a statement relating the two by means of an isomorphism. Abstract Algebra instructors sometimes call the 1st-IT "the kernel-image theorem". On the contrary, these ostensives are absent ${ }^{6}$ from van der Waerden's statement who also contains a reciprocal and expresses a characterisation of homomorphic images in terms of quotient groups. The "law of homomorphism" therefore establishes the rationale of both homomorphisms, as a generalisation of isomorphisms (later, van der Waerden will use the locution "many-one isomorphism" which is very illuminating), and quotient groups. The second ${ }^{7}$ and third ${ }^{8}$ isomorphism theorems for groups, called "the two laws of isomorphism", are introduced in chapter 6, section 46 of the book, before studying composition series, as they are seen as the main tools in order to establish the important theoretical result known as Jordan-Hölder Theorem.

On the contrary, the main ostensives in the discussion of homomorphisms by Artin are the function, the kernel and the image: "every group homomorphism $\varphi$ determines two important subgroups: its image and its kernel [...] In addition to being a subgroup, the kernel has a special property which is subtle but very important: [...] it is a normal subgroup". The 1st-IT is stated

\footnotetext{
${ }^{5}$ Chevallard distinguishes ostensive objects, explicitly designated by the teacher, and nonostensive objects which remain implicit but play a role in the execution or justification of a mathematical technique; ostensives and non-ostensives emerge together and are co-activated, but Chevallard argues that a mathematical activity is sensitive to ostensives which play a semiotic role.

${ }^{6}$ The word "image" in vernacular language has not the same status as the symbolic notation $\Im m$ which isn't needed in van der Waerden's case since a homomorphism is onto. The importance of kernels and images in modern expositions is related to the development of homological algebra.

${ }^{7} K H / H \simeq K /(K \cap H)$, where $K, H$ are two subgroups of $G$ and $H$ is normal.

${ }^{8} G / K \simeq(G / H) /(K / H)$ where $H, K$ are normal subgroups of $G$, and $K$ contains $H$.
} 
and proved (theorem 10.9), followed by the comment that "This is our fundamental method of identifying quotient groups". The following generalisation of the 1st-IT, which did not appear in van der Waerden's textbook, is introduced in chapter 6 as a tool to "work freely with the concept of generators and relations":

Proposition 1 (Mapping property of quotient groups) Let $N$ be a normal subgroup of $G$, let $\bar{G}=G / N$, and let $\pi$ be the canonical map $G \rightarrow \bar{G}$ defined by $\pi(a)=\bar{a}=a N$. let $\varphi: G \rightarrow G^{\prime}$ be a homomorphism whose kernel contains $N$. There is a unique homomorphism $\bar{\varphi}: \bar{G} \rightarrow G^{\prime}$ such that $\bar{\varphi} \pi=\varphi$ :

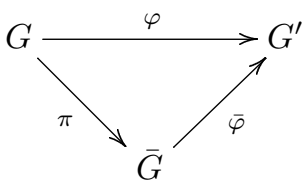

This map is defined by the rule $\bar{\varphi}(\bar{a})=\varphi(a)$.

The condition on the kernel is also necessary to obtain such a factorisation $\bar{\varphi} \pi=\varphi$ but it isn't stated in the proposition since the goal pursued by Artin, who keeps to his philosophy, is not to study the conditions of a formal property of factorisation but to apply the formalisation to concrete problem-solving. This proposition also introduces a new type of semiotic representation: a diagrammatic representation involving symbols and arrows, in the spirit of homological algebra in which diagrammatic reasoning ("diagram chasing"), as a part of the standard mathematical practise, takes advantage of the geometric possibilities offered by the two-dimensional sheet of paper. Although the representational more than the computational aspect of this practise is involved in the above proposition, the diagram may play an important cognitive role in the encapsulation of the different objects considered in the proposition into an integrated whole. The second and third isomorphism theorems for groups are left as exercises at the end of the chapter, as applications of the 1st-IT. They are not presented in the main text since the author does not give any account of Jordan-Hölder's theorem which may be regarded as an essentially theoretical result.

In Guin (1997), quotient groups are introduced independently of the notion of morphism, in a more general context: the problem is to determine, for a general equivalence relation $\mathcal{R}$ on a set $E$ endowed with a composition law, the conditions for this law to induce on the set of equivalence classes $E / \mathcal{R}$ again a composition law. The answer is the following: $\mathcal{R}$ must be compatible ${ }^{9}$ with the law; when $E$ is a group $G$, the relation $\mathcal{R}$ must coincide with that defined by a normal subgroup $H$ of $G$ so that equivalent classes are cosets. As we can see, the correspondence "normal subgroup - quotient - homomorphism" put forward by van der Waerden is not the principle of organisation of the theory for Guin who grounds his exposition on general set-theoretic constructs: normal subgroups are not distinguished as kernels of homomorphisms

${ }^{9} \forall x, x^{\prime}, y, y^{\prime} \in E,\left[x \mathcal{R} x^{\prime}\right.$ and $\left.y \mathcal{R} y^{\prime}\right] \Rightarrow\left[x y \mathcal{R} x^{\prime} y^{\prime}\right]$ 
but because this is the good notion to build quotients in general in Group Theory. Guin introduces a generalisation of proposition 1, entitled "théorème de passage au quotient" (TPQ), whose purpose is to establish the conditions for a morphism $f: G \rightarrow G^{\prime}$ to induce a morphism $\bar{f}: G / H \rightarrow G^{\prime} / H^{\prime}$ between quotients which "commutes" with quotient maps. The second and third isomorphism theorems are stated (without any name or numbering) and proved as applications. Although the TPQ theorem is more demanding in terms of conceptualisation since quotients are considered on both sides, it appears as the appropriate formalisation for the construct of an isomorphism between two quotients. Nevertheless, such applications are also workable using proposition 1 and most of the cases assigned to students are of this form. Guin and Artin therefore give different answers to a tension between the mathematical pertinence of formalisations and ecological and cognitive concerns.

\subsection{Thematisation}

In Ring Theory, as a second algebraic structure studied, all three authors present homomorphisms first.

Van der Waerden defines the concept of ideal of a ring with the motivation that ideals are "subrings which play a special role, in analogy to the normal divisors in group theory". The goal of section 16, chap. 3, is to develop the formal analogy between the group and ring contexts, which leads to the statement of the "law of homomorphism for rings" as a characterisation of homomorphic images of ring homomorphisms in terms of residue class rings. Moreover, "the transition to the residue class ring transforms congruences into equalities" which appears as a motivation for the construction of quotient rings related to the simplification of computational tasks. Laws of isomorphism (the second and third theorem) for rings are not stated in (Waerden, 1930), probably due to the lack of standard applications for these results. Therefore, the thematisation which is guiding van der Waerden's exposition is also balanced by the consideration of the specific backgrounds of Group Theory and Ring Theory, each having its set of questions and problems.

According to Artin, the "most important ring homomorphisms are those obtained by evaluating polynomials", together with the unique homomorphism $\mathbb{Z} \rightarrow A$, probably because these homomorphism lead to the definition of minimal polynomials and the characteristic of a ring $A$ respectively. For Artin, the rationale of the homomorphism concept therefore resides, rather than in the formalisation of a relation of comparison between two objects in abstract theories of structures, in the further development of results concerning concrete mathematical objects. He states that the kernel of a ring homomorphism $\varphi$ is "defined in the same way as the kernel of a group homomorphism" and possesses a special property, apart from being closed under the ring operations, which is "abstracted in the concept of an ideal". The terminology is attributed to Dedekind and the reader is referred to chapter 11 for further details on the historical origin of ideals. The principality of $\mathbb{Z}$ and $K[x]$ is established: again, 
Artin does not focus on the formal development of Ring Theory in analogy to Group Theory but aims at substantial results concerning concrete objects.

In the construction of quotient rings and its connection to the homomorphism concept, the corollary that ideals are kernels of homomorphisms, is not stated: thematisation is visible in the statements and proofs given by Artin, as a principle of economy, not in a meta-discourse whose aim would be to underpin the formal analogy between both theories as an anchor point to its development. Artin points out that "the proof has already been carried out in the special case that $R$ is the ring of integers" and that "if we forget about multiplication and consider only the addition law, the proof has already been given", building both on the paradigmatic example, thus idealisation, and on thematisation. The mapping property of quotient rings is stated as well as the 1st-IT for rings as its corollary. The proof is left to the reader with the argument that it is an easy thematisation ("as in chapter 6 and chapter 2 , on can show the following"). The third isomorphism theorem, denominated "Correspondence Theorem", is stated and proved: the statement insists on the correspondence between ideals of a ring $R$ and ideals of the quotient $\bar{R}=R / I$, whence the name, which is much more telling than the numbering that often appears in the literature. The second isomorphism theorem is left as an exercise at the end of the chapter, since it won't find any application in the sequel. Artin's meta-discourse on quotient rings is not directed towards idealisation and thematisation, but it underlines that "the quotient construction has an important interpretation ${ }^{10}$ in terms of relations among elements in a ring $R$ ".

Following his formal account, Guin brings out the special property defining an ideal as the condition for the relation $\mathcal{R}$ on a ring to be also compatible with the multiplicative law. The TPQ is stated in the ring context with a short proof indicating that $\bar{f}$ is already defined as a group morphism so that "it suffices to verify that it is a morphism of rings, which is immediate". The correspondence between ideals of a quotient and ideals of the ring is stated and proved in the more general context of two rings $A$ and $B$ related by an onto-morphism $f: A \rightarrow B$, as well as the third isomorphism theorem which connects the resulting quotients. The second isomorphism theorem is not stated, probably for a lack of application. Although Guin's book conveys the structural image of algebra, thematisation appears more as a principle of economy, building on the fact that rings are groups in particular, than a principle that should prescribe, both mathematically and didactically, the writing of the theory. Idealisation and generality as a principle to manifest the inner reasons for a mathematical result to hold presides towards thematisation in the didactical choices of Guin manifested in mathematical statements, mathematical organisations and the meta-discourse.

\footnotetext{
${ }^{10}$ The point is that "the quotient ring $\bar{R}=R / I$ should be viewed as the ring obtained by introducing the $n$ relations $a_{1}=0, \ldots, a_{n}=0$ into $R$ ", where $I$ is generated by the $a_{i}$ 's. According to the third-IT, introducing a relation $\bar{b}=0$ into the ring $\bar{R}=R /(a)$ amounts to "killing $a$ and $b$ at the same time": $\bar{R} /(\bar{b}) \approx R /(a, b)$.
} 


\section{A classroom experiment on ring-homomorphisms}

As announced in the introduction, I will present in this section the piece of didactical engineering that has been produced on the basis of the preceding analyses. The goal is the teaching of ring-homomorphisms to students who are already acquainted with group-homorphisms, thus involving the phenomenon of thematisation, and with a view to using the meta lever. I will explain in the next section the choices that I made in the construction of my situation (Brousseau, 1997), in particular how I took into account the preceding elements which already contributed to break the illusion of transparency concerning the way mathematicians thematise the homomorphism concept. Following the methodology of didactical engineering (Artigue, 2009), the process of validation of the hypotheses underlying the design is internal. I will thus give an a priori analysis of the tasks before presenting the empirical data obtained through a classroom experimentation, and the a posteriori analysis will be about contrasting this data with the reference created by the a priori analysis.

\subsection{Context and statement of the tasks}

I was in charge of a course in Ring and Field Theories at Montpellier University, for third year students in pure mathematics having had a first course in Group Theory during the preceding semester. This was an opportunity to test teaching material that I developed on the basis of the epistemological analyses presented here, by endorsing both the role of the teacher and the researcher.

I chose to introduce homomorphisms during the lectures as structurepreserving functions, emphasising the condition ${ }^{11} f(1)=1$. To facilitate the retrieval, the ring data was denoted $(A,+, \cdot, 1)$. Following Guin $(1997)$, the general idea was developed that homomorphisms aimed at "comparing" rings: if $f: A \rightarrow B$ was bijective then $A$ and $B$ were "essentially the same" and could be identified. If it was only injective (1-1), then $A$ could be identified with a subring of $B$ and the kernel measured the defect of injectivity, if it was surjective (onto), then $B$ could be identified with a quotient of $A$ through the first isomorphism theorem. I chose to adopt the level of generality of M. Artin (1991) (the mapping property of quotients compared to the TPQ); as an application of proposition 1 in the ring context, which was stated with a necessary and sufficient condition $I \subset \operatorname{ker} f$ (unlike Artin), presented as a tool for constructing homomorphisms from quotient rings and denominated explicitly "factorisation theorem for ring homomorphisms", I proved that $\mathbb{Z} \rightarrow \mathbb{Z} / 2 \mathbb{Z}$ factored through $\mathbb{Z} / 4 \mathbb{Z}$ and induced an isomorphism $(\mathbb{Z} / 4 \mathbb{Z}) /(\overline{2}) \simeq \mathbb{Z} / 2 \mathbb{Z}$. The lecture carried on with the third isomorphism theorem which generalised such isomorphisms and were called "simplification theorem for quotients of quotients" (acknowledging that $\overline{2}=2 \mathbb{Z} / 4 \mathbb{Z}$ ). To summarise the didactic intent, the tool-object dialectic

\footnotetext{
${ }^{11}$ which wasn't automatic unlike $f(0)=0$, since $A^{*}$ was not in general a group for the multiplicative law
} 
(Douady, 1986) was at play and epistemological insight was given to connect the formalism with cognitive processes of comparison and identification. The examples taken were the arithmetic groups $\mathbb{Z} / n \mathbb{Z}$ as paradigmatic examples of quotient groups.

The tasks proposed to students in order to practise this theoretical content were the following.

1. Recall the definition of a ring homomorphism $f: A \rightarrow B$.

Whenever $f$ is an isomorphism, one often identifies $A$ and $B$. Which identification can we make when $f$ is only injective (resp. surjective)?

2. Recall the factorisation theorem of a homomorphism $f: A \rightarrow B$ through a quotient $A / I$ and give a proof.

3. Can $\mathbb{Z} / 4 \mathbb{Z}$ be identified with a subgroup of $\mathbb{Z} / 8 \mathbb{Z}$ ? Justify your answer.

4. Can $\mathbb{Z} / 4 \mathbb{Z}$ be identified with a subring of $\mathbb{Z} / 8 \mathbb{Z}$ ? Justify your answer.

5. Construct a ring-homomorphism that links $\mathbb{Z} / 4 \mathbb{Z}$ and $\mathbb{Z} / 8 \mathbb{Z}$.

6. Can $\mathbb{Z} / 4 \mathbb{Z}$ be identified with a quotient ring of $\mathbb{Z} / 8 \mathbb{Z}$ ? Justify your answer.

7. Let $A$ be a ring. Show that there exists a unique ring-homomorphism $\mathbb{Z} \rightarrow$ A.

8. Under which hypothesis on the integers $n$ and $m$ does there exist a ringhomomorphism $\mathbb{Z} / n \mathbb{Z} \rightarrow \mathbb{Z} / m \mathbb{Z}$ ? In this case, is the homomorphism unique?

The didactical stake of this exercise, regarded as a situation in the framework of the Theory of Didactical Situations (Brousseau, 1997), is the notion of homomorphism as a tool to "compare" different rings of the form $\mathbb{Z} / n \mathbb{Z}$ in terms of subrings and quotients. Regarding thematisation of proposition 1, it was expected that students' practise of factorising homomorphisms through quotient groups would help them to do so in the ring context. Homomorphisms might be constructed by hand in the case of small cardinals ( $n$ as a didactic variable) whereas in the last question the use of the factorisation theorem was quite convenient. In a concrete-abstract dialectic, the idea was that students might be able to realise that residue classes mod 8 refined classes $\bmod 4$ since $8 \mathbb{Z} \subset 4 \mathbb{Z}$ and therefore $x \bmod 8 \mapsto x \bmod 4$ defined a homomorphism $\mathbb{Z} / 8 \mathbb{Z} \rightarrow \mathbb{Z} / 4 \mathbb{Z}$. They should do so as they connected this concrete case to proposition 1 (and its proof) which was both a general and abstract formalisation of this phenomenon (object aspect) and a tool to write down, justify and control the conditions of existence of such a homomorphism (tool aspect). Indeed, any homomorphism $\mathbb{Z} / n \mathbb{Z} \rightarrow \mathbb{Z} / m \mathbb{Z}$ came from a homomorphism $\mathbb{Z} \rightarrow \mathbb{Z} / m \mathbb{Z}$ (uniquely determined in question 7 ) by application of the factorisation theorem, which made the universality of such a procedure visible. I therefore made the hypothesis that this situation was pertinent to impart both the rationale of the homomorphism concept and the factorisation theorem, which would be evaluated by an analysis of the empirical data that was collected in the classroom realisation phase of the engineering.

Let us conclude this section by explaining how the milieu (Brousseau, 1997) was set up. The 20 students worked in small groups (3-5 students). They were not allowed to read my lecture notes that I had distributed in the form of handouts, but could call me whenever needed. The pedagogical 
strategy was that interactions with peers would favour the discussion among students of the elements of meta-discourse that was introduced in the milieu (the epistemological idea of identifications), thus contribute to the functioning of the meta-lever. Moreover, the teacher was in a position of giving helpful feed-back in order to control the effect of the meta-discourse. Two sessions of 1.5 hours were dedicated to the activity, half of the second session being used for the correction that I wrote on the blackboard in order to institutionalise the new knowledge produced.

\subsection{A priori analysis}

The retrieval of the definition of a homomorphism was expected to be correct, except a missing $f(1)=1$ or extra non-necessary $f(0)=0$ condition which might be interpreted as a lack of understanding of the raison d'être of the former condition. Solving question 1 required to translate the task in the following terms: "what kind of isomorphism does $f$ induce when it is injective (resp. surjective)". The idea of identifying rings was a motivation for the homomorphism concept in view of the answers to question 1 , which had been explicitly written down in the handouts, but it might also be a draw-back during the completion of the task which was not stated in standard mathematical terms. The conceptualisation of 1st-IT as a procedure to transform a homomorphism (the "many-to-one isomorphism") into an isomorphism was the main obstacle to question 1. Nevertheless, the injectivity of $f$ produced a "degenerate case" in which $A$ was directly isomorphic to $f(A)$, so that it might lead to a blockage. Independently of 1st-IT, an injective homomorphism might be conceptualised as an embedding $A \simeq f(A) \subset B$ which generalised the inclusion map. Other students might therefore be capable of answering the case $f$ injective but get stuck on the case $f$ surjective. It would be worth observing whether students express their findings in terms of identifications with subrings and quotients in vernacular language or use only mathematical signs.

Group-work was expected to be beneficial to the retrieval and proof of the factorisation theorem (question 2) which was quite a formal and conceptually demanding task: the case of an individual examination in limited time would certainly produce different results. The necessary and sufficient condition $I \subset \operatorname{ker} f$ might be stated as sufficient only, which corresponded to the standard use of the theorem (but the reciprocal would be used in question 8). The necessity was also connected to the understanding of the raison d'être of this condition: elements of $I$ mapped to 0 on the quotient $A / I$, therefore to 0 in $B$ since $\bar{f}$ was a homomorphism. This was a simple case of "diagram chasing" discussed in section 4.3: it would be worth observing the presence of such a representation on the students' sheets and analysing the role this might play in their procedures. A common mistake, referring to my experience, was the inversion of the inclusion ( $\operatorname{ker} f \subset I$ ) that I could often observe in examination papers. It should be noted that students had no semantic control on this condition: a simple instantiation of the theorem would be of the type 
that was being constructed in this exercise. With regard to the proof, the difficulty of articulating $\psi$ and $\phi$ (see theorem 1) identified by Nardi (2000) was greatly taken in charge didactically by the formulation: " $f$ factors through the quotient and induces $\bar{f}$ ". The list of properties that needed to be checked included the well-definedness of $\bar{f}$ and the homomorphic properties. In general, Abstract Algebra instructors insisted heavily on the fact that it needed to be proved that a map, defined on a quotient by a formula involving a representative of a class, did not depend on the choice of the representative. If all the attention was drawn towards this property, students might forget to check the homomorphic property which was a direct consequence of the fact that $f$ was itself a homomorphism.

Question 3 might be solved using the factorisation theorem for groups: the map $\mathbb{Z} \rightarrow \mathbb{Z} / 8 \mathbb{Z}$ defined by $x \mapsto \overline{2 x}$ had $4 \mathbb{Z}$ as kernel and therefore defined an embedding $\mathbb{Z} / 4 \mathbb{Z} \hookrightarrow \mathbb{Z} / 8 \mathbb{Z}$. The formula $f(x)=\overline{2 x}$ might be discovered reflecting on the condition ker $f=4 \mathbb{Z}$ and the formula $f(x)=x f(1)$ since 1 generated $\mathbb{Z}$ as an additive group. But it should be pointed out that it was more convenient to solve the problem by realising that it amounted to deciding whether $\mathbb{Z} / 8 \mathbb{Z}$ contained an element of order 4 or not. As it was easily checked that there were 2 elements $( \pm \overline{2})$ of order 4 , we saw that there were 2 different ways of identifying $\mathbb{Z} / 4 \mathbb{Z}$ with a subgroup of $\mathbb{Z} / 8 \mathbb{Z}$. This was consistent with the structure theorem of cyclic groups which might be invoked by students and which asserted that $\mathbb{Z} / n \mathbb{Z}$ contained, for every divisor $d$ of $n$, a unique subgroup of order $d$, which was also cyclic. Students might also apply the "correspondence-theorem" which described the subgroups of the quotient $\mathbb{Z} / 8 \mathbb{Z}$ in terms of subgroups of $\mathbb{Z}$ containing $8 \mathbb{Z}$. As we could see, there were different theoretical tools available in Group Theory to solve that question. It would be worth observing whether such tools would actually be used by students or if they would engage in building an isomorphism by hand. An error which might be predicted is the consideration of the subset $\{\overline{0}, \overline{1}, \overline{2}, \overline{3}\}$ of $\mathbb{Z} / 8 \mathbb{Z}$ as a group by students who looked for a straightforward identification.

Question 4 might be solved using question 3: a ring-homomorphism was also a group-isomorphism but the two group-homomorphisms did not verify the $f(1)=1$ condition. Students might also try to construct a homomorphism $\mathbb{Z} / 4 \mathbb{Z} \rightarrow \mathbb{Z} / 8 \mathbb{Z}$, beginning with $f(\overline{1})=\overline{1}$, and understand that it was completely determined. The contradiction came from the fact that $\overline{4}=\overline{0}$ mapped to $\overline{4}$ which wasn't $\overline{0}$ in $\mathbb{Z} / 8 \mathbb{Z}$. Students might nevertheless be confused if they used the same sign for residue classes in both rings as I have just done. Another strategy would be to determine the subrings of $\mathbb{Z} / 8 \mathbb{Z}$ using the fact that it was generated by $\overline{1}$ as a ring and that a subring contained $\overline{1}$ by definition. This strategy might be suggested by analogy to the group context but, unlike cyclic groups, we saw that $\mathbb{Z} / n \mathbb{Z}$ contained no proper subring, which settled the question.

In question 5, students might have learnt from question 4 that such a homomorphism should not be constructed in the form $\mathbb{Z} / 4 \mathbb{Z} \rightarrow \mathbb{Z} / 8 \mathbb{Z}$, be it injective or not, but the other way round, which might appear at first sight unnatural. The homomorphism was again totally determined by $f(\overline{1})=\dot{1}$, so 
that $f(\bar{x})=\dot{x}$, and it amounted to proving rigorously that this defined a homomorphism, which was straightforward. It should also be checked that this formula did define a map, which was likely to be omitted by a few students. The didactical intent was that students might realise that they were in fact checking, in a particular instance, the same properties that allowed the general construction of $\bar{f}$ in question 2 and concluded that this theorem is a convenient formalisation that saved a lot of verifications. Although it was possible that students used directly the theorem, it was likely that they would favour methods that were less conceptually demanding, the more so as the rings presented are very elementary and the students were in a learning stage in relation to the homomorphism machinery.

Solving question 6 amounted to noticing that the previous map was surjective (since it was induced by factorisation of a surjective map) in order to conclude using question 1.

The uniqueness in question 7 should be easy, since students had already used the $f(1)=1$ property in a context in which the ring was generated by its unit element. A formal writing $f(n)=n 1_{A}$ as was standard in abelian groups might facilitate the proof of existence, but students might be unaware that it actually amounted to assuming that this defined a group-homomorphism. They might also be confused when such a notation was combined with the multiplication law in $A$, and therefore make the following improper use of symbolic manipulations: $n 1_{A} \cdot m 1_{A}=n m 1_{A} \cdot 1_{A}=n m 1_{A}$.

Again, $\mathbb{Z} / n \mathbb{Z}$ was generated by its unit, so that students should easily settle the uniqueness of a homomorphism $\mathbb{Z} / n \mathbb{Z} \rightarrow \mathbb{Z} / m \mathbb{Z}$ and focus on studying the conditions for this map to define a homomorphism. The case of question 5 was likely to orient them on the condition " $m$ divides $n$ " as a conjecture obtained by intuitive induction. Unlike question 5 , the generality of the situation (it was more difficult to figure out how residue classes modulo $n$ mapped to classes modulo $m$ in general) and the presence of question 7 might suggest the use of the factorisation theorem. Students should also know from the recent lecture on ideals that an inclusion of principal ideals $(a) \subset(b)$ was equivalent to the condition " $b$ divides $a$ ", which would allow them to connect easily the hypothesis of the theorem and the divisibility condition. In order to demonstrate that this condition was necessary, the didactical intent, as was already pointed out, was that students should realise that any such homomorphism was obtained by application of the theorem, and thus use the uniqueness of question 7 together with the necessary condition in the statement of the theorem. This was nevertheless quite demanding on the point of view of logical reasoning. Moreover, students might also generalise the argument, used in question 4, that $\bar{n}=\overline{0}$ mapped to $\dot{n}=\dot{0}$ in $\mathbb{Z} / m \mathbb{Z}$, whence $m$ divided $n$. As was often the case in algebra, the existence of an elementary solution might spoil the conceptual point of view that the exercise was aiming at. 


\subsection{A posteriori analysis}

The 5 groups of students are designated S1 to S5 in the sequel. The students worked with instructions to write down their answers on a sheet, even if it was incomplete, and to keep track of their reasoning, even if looked like a dead-end track. The sessions were audio-taped, unfortunately with a single recorder that I carried with me as teacher-researcher. The sheets from the 5 groups and the recording constitute the data that will be analysed below, by comparison with the a priori analysis according to the methodology of didactical engineering.

All the groups gave a correct definition of a ring-homomorphism, except S2 who wrote $f\left(e_{A}\right)=e_{B}$ and mentioned "identity element of $B$ " without indicating which type of identity element (multiplicative or additive) was meant. All the other groups wrote down " $f\left(1_{A}\right)=1_{B}$ ". It had been underlined during the lectures that the multiplicative identity of a ring was called the unity, the notation $e$ being generic for any composition law. Two of the groups did not distinguish the laws of the rings $A$ and $B$, two groups used indices $\left(+_{A}\right.$ and $\times_{A}$ ), one group denoted the second $\operatorname{ring}(B, *, \Delta)$. The second part of question 1 , which concerned the identifications to be made whenever $f$ was injective or surjective, caused much trouble to the students. Only S4 gave a complete and correct answer (see table 1). All the other groups seemed to be confused between identifications on the basis of an isomorphism and equalities. This raised the issue of the conceptualisation of identifications under an equivalence relation such as the isomorphic relation: one could make the hypothesis that the lack of an adequate semiotic mediation hindered such a conceptualisation. Indeed, such an act of abstraction led, in semiotic terms, to the replacement of the equivalence relation by an equality, equivalence classes being conceptualised as single objects, for instance $\frac{1}{3}=\frac{2}{6}$. In Group Theory, mathematicians wrote $G_{1} \simeq G_{2}$ (or $\left.G_{1} \cong G_{2}\right)$ : if they did think of the two groups as one and the same on an algebraic point of view, they would never write $\bar{G}_{1}=\bar{G}_{2}$. Dually, the equality was seldom given by students as an example of an equivalence relation (Durand-Guerrier et al., 2015). A lack of understanding of the relationship between equivalence relations and equalities in connection with the very rationale of equivalence relations appeared therefore as an obstacle against the completion of the task. In the experiment, groups S1 and S2 looked for equalities rather than isomorphisms. The first-IT was used only by the group S4: this suggested that the rationale of this theorem as a procedure to convert homomorphisms into isomorphisms was not integrated by most of the students. On the contrary, the answers of group S5, which might be interpreted as a vague application of a principle of symmetry between the two cases, showed a lack of control in relation to basic notions of set theory: $f^{-1}(B)$ coincided with $A$ when $f$ was surjective, and therefore couldn't be isomorphic to $B$ when $f$ was not injective.

The statement of the factorisation theorem (question 2) was retrieved properly by the 5 groups. The group S4, which happened to comprise the best achieving students (these decided to team), was the only one who did not reproduce the diagram which illustrated the relation $f=\bar{f} \circ \pi$. As was expected 
Table 1 Answers to question 1: identifications on the basis of isomorphisms

\begin{tabular}{lll}
\hline group number & $f$ injective & $f$ surjective \\
\hline S1 & $A \cong f(A)($ we also have ker $f=\{0\})$ & we only have $f^{-1}(B)=A$ \\
S2 & $\operatorname{Im} f=B$ & ker $f=\{0\}$ \\
S3 & - & \\
S4 & one identifies $A$ with $f(A)$ & one identifies $B$ with $A /$ ker $f$ \\
S5 & $A \simeq f(A)$ & $B \simeq f^{-1}(B)$ \\
\hline
\end{tabular}

in the a priori analysis, the condition on the ideal was not stated as necessary by a few students (groups S2 and S4) until the point had been discussed with the teacher. The groups S1, S2 and S3 did not check the homomorphic property, so that $\mathrm{S} 4$ was the only one to give a complete proof. The group S5 did not write down any proof at all, even after having recapitulated with the help of the teacher the different points that needed to be checked. A student argued that it was too deep ("we need to think hard").

The answers to question 3 given by the different groups confirmed the availability of a diversity of techniques, including advanced results in Group Theory: S1 used the structure theorem for cyclic groups, S2 brought froward the subset $\{\overline{0}, \overline{2}, \overline{4}, \overline{6}\}$ and asserted that it was isomorphic to $\mathbb{Z} / 4 \mathbb{Z}$ by indicating the orders of elements. The group $\mathrm{S} 4$ constructed by hand an injective map $\mathbb{Z} / 4 \mathbb{Z} \rightarrow \mathbb{Z} / 8 \mathbb{Z}$ and claimed that it was trivial to check that it was an homomorphism. They also commented that "since $\mathbb{Z} / 8 \mathbb{Z}$ possesses a cyclic subgroup of order 4 , it suffices to send generators to generators": the conceptual point of view emerged after a concrete manipulation of objects. The group S3 wrote: " $\mathbb{Z} / 4 \mathbb{Z}=\{\overline{0}, \overline{1}, \overline{2}, \overline{3}\}, \mathbb{Z} / 8 \mathbb{Z}=\{\overline{0}, \overline{1}, \ldots, \overline{7}\}, \mathbb{Z} / 4 \mathbb{Z} \subset_{\text {subgroup }} \mathbb{Z} / 8 \mathbb{Z}$ because $n \mid m \Rightarrow m \mathbb{Z} \subset n \mathbb{Z} \Rightarrow \mathbb{Z} / m \mathbb{Z} \supset \mathbb{Z} / n \mathbb{Z}$ ". The designation of residue classes modulo 4 and 8 by the same sign brought the students to the illusion that $\mathbb{Z} / 4 \mathbb{Z}$ was naturally included in $\mathbb{Z} / 8 \mathbb{Z}$.

A discussion between the students of S3 and the teacher followed: the latter pointed out that these were two different types of classes and hid the sign " $\mathbb{Z} / 4 \mathbb{Z}=$ " in the students' expression.

TEACHER: So, is the set $\{\overline{0}, \overline{1}, \overline{2}, \overline{3}\}$ of classes $^{12}$ modulo 8 a subgroup of $\mathbb{Z} / 8 \mathbb{Z}$ ?

A: Yes

TEACHER: Let's see: we have $\overline{1}+\overline{2}=\overline{3}, \overline{1}+\overline{3}=\overline{4}$, which isn't $\overline{0}$, this comes out of the

set.

A: Hum... the set is not stable, so it isn't a subgroup...

TEACHER: Yes! But there might exist another subgroup isomorphic to $\mathbb{Z} / 4 \mathbb{Z}$...

A: Hum... I cannot see any.

TEACHER: Let us think abstractly. $\mathbb{Z} / 4 \mathbb{Z}$ is a cyclic group; so, what do we need to construct such a group?

A: A generator

\footnotetext{
${ }^{12}$ I actually said : "class of zero, one, two, three". In the sequel, students also referred to $\bar{x}$ as "class of $\mathrm{x}$ " most of the time, the exceptions being obvious abbreviations in case multiple classes were mentioned consecutively.
} 
With the help of the teacher, the students looked for an element of order 4 in $\mathbb{Z} / 8 \mathbb{Z}$, which led to the set $\{\overline{0}, \overline{2}, \overline{4}, \overline{6}\}$. At this stage, the teacher left to respond to another group but the discussion among S3 was still recorded.

B: Why did he hide $\mathbb{Z} / 4 \mathbb{Z}$ ? I don't understand

A: He calculates in $\mathbb{Z} / 4 \mathbb{Z}$ but he is always in $\mathbb{Z} / 8 \mathbb{Z} . \overline{1}+\overline{3}=\overline{0}$ in $\mathbb{Z} / 4 \mathbb{Z}$ but $\overline{4}$ in $\mathbb{Z} / 8 \mathbb{Z}$.

B: O.K.

A: So we have the set $\{\overline{0}, \overline{2}, \overline{4}, \overline{6}\}$.

C: $\overline{4}+\overline{6}=\overline{10} \ldots \overline{10}=\overline{2}$ in $\mathbb{Z} / 8 \mathbb{Z}$. It works.

A: But does it really prove that $\mathbb{Z} / 4 \mathbb{Z}$ may be identified with a subgroup of $\mathbb{Z} / 8 \mathbb{Z}$ ?

B: It isn't a subgroup.

C: We need to talk about isomorphisms at some point...

A: This question is puzzling me...

These discussions showed how much students were dependant on the semiotic representation $\{\overline{0}, \overline{1}, \overline{2}, \overline{3}\}$ of the group $\mathbb{Z} / 4 \mathbb{Z}$, which wasn't conceptualised as a model of an abstract group (the cyclic group of order 4 ). They looked for an inclusion rather than an embedding. The set $\{\overline{0}, \overline{1}, \overline{2}, \overline{3}\}$ was not a subgroup of $\mathbb{Z} / 8 \mathbb{Z}$, therefore students could hardy figure out that there did exist a subgroup isomorphic to $\mathbb{Z} / 4 \mathbb{Z}$. Although student $\mathrm{A}$ knew that a cyclic group was generated by a single element, she did not recognise that $\{\overline{0}, \overline{2}, \overline{4}, \overline{6}\}$ is isomorphic to $\mathbb{Z} / 4 \mathbb{Z}$. For the student $B, \mathbb{Z} / 4 \mathbb{Z}$ still coincided with $\{\overline{0}, \overline{1}, \overline{2}, \overline{3}\} \ldots$ which wasn't a group. The isomorphism as an equivalence relation aiming at abstracting algebraically-equivalent groups was not yet acquired by this group.

The group S5 introduced yet another method which was not predicted in the a priori analysis and permitted to embed both groups $\mathbb{Z} / 4 \mathbb{Z}$ and $\mathbb{Z} / 8 \mathbb{Z}$ in a common framework, so that the former was included in the latter: the students wrote " $\mathbb{Z} / n \simeq\left(\mathrm{e}^{2 i \pi k / n}, \times\right),\left(\mathrm{e}^{2 i \pi k / 4}\right) \subset\left(\mathrm{e}^{2 i \pi k / 8}\right)$ and $\times_{\mathbb{Z} / 4}=\times_{\mathbb{Z} /\left.8\right|_{\mathbb{Z} / 4}}$ and $e_{\mathbb{Z} / 8} \in \mathbb{Z} / 4$. Therefore $\mathbb{Z} / 4<\mathbb{Z} / 8$." The students' notations showed that they were identifying $\mathbb{Z} / n \mathbb{Z}$ with the model in the unit circle, and therefore considered $\mathbb{Z} / 4 \mathbb{Z}$ as a subgroup of $\mathbb{Z} / 8 \mathbb{Z}$, thus making no distinction between an inclusion and an embedding. In semiotic terms, the sign $\mathbb{Z} / n \mathbb{Z}$ designated both the group of integers modulo $n$ and this other representative of the isomorphic class of this group. This indicated that these students had built an abstract group concept but the drawback was that the mathematical distinction between an isomorphism and an equality was not maintained. The didactical strategy of motivating the isomorphism and homomorphism concepts by means of identifications was thus encountering an obstacle that had not been anticipated: we saw in question 1 that many students related identifications to equalities; it was probable that the sentence "we can identify isomorphic groups" was interpreted by students as an invitation to take as equal representatives whereas only classes were equal.

The group S1 did not attack question 4 (and any of the following questions) whereas S2 and S4 solved the question with the argument that a subring of $\mathbb{Z} / 8 \mathbb{Z}$ contained $\overline{1}$, which generated the ring. On the contrary, S3 was in favour of a positive answer and wrote down the subring criterion that needed to be checked. This group couldn't deduce from the stability conditions and the presence of $\overline{1}$ that such a subring was the full ring without the help of the teacher, therefore leading to the opposite conclusion. The group S4 began by 
considering the multiplicative structure of the rings $\mathbb{Z} / 4 \mathbb{Z}$ and $\mathbb{Z} / 8 \mathbb{Z}$, thus the groups of units. The help of the teacher was needed to correct the cardinals of these groups, as they obtained erroneous results, but the strategy proved to be ineffective in order to demonstrate the students' intuition that the answer was negative. The teacher thus asked if the group obtained in question 3 was a ring. The change of frameworks from the roots of unity to residue classes of integers operated by students led to the consideration of the inclusion $\{\overline{0}, \overline{1}, \overline{2}, \overline{3}\} \subset$ $\{\overline{0}, \overline{1} \ldots, \overline{7}\}$. Another intervention of the teacher was needed to point out that the former was not a ring, therefore indicating a mistake in the conversion. The set $\{\overline{0}, \overline{2}, \overline{4}, \overline{6}\}$ emerged and the students checked that it was closed under both laws. The property that a subring was endowed with the same unity as the ring had not been integrated by this group. Further interventions of the teacher was needed to question a "missing axiom". Distributivity and identity elements were discussed before coming to the unity. The students then realised immediately that this element generated the full ring.

Most of the groups constructed the homomorphism $\mathbb{Z} / 8 \mathbb{Z} \rightarrow \mathbb{Z} / 4 \mathbb{Z}$ by hand, by setting $\bar{x}^{4} \mapsto \bar{x}^{8}$, where the exponent indicated the kind of residue class under consideration. This is consistent with the a priori analysis; only S3 and S4 applied the factorisation theorem, and S4 did it second hand. Moreover, S2 wrote $\bar{x} \mapsto \bar{x} \bmod 4$, which was incorrect since $\bar{x} \bmod 4$ did not make sense. As this was pointed out by the teacher, a student successfully corrected by writing $\bar{x} \mapsto \overline{x \bmod 4}$. The students understood that the well-definiteness of the map needed to be checked. All the groups that solved question 5 also successfully solved question 6 by application of the 1st-IT.

Question 7 was solved by groups S2, S4 and S5 but only S4 managed to connect the multiplicative property of the homomorphism with the distributive property in the ring $A$. The other groups wrote $f(x)=x 1_{A}$ without further detail on the meaning of such a writing, which led to the erroneous proof " $x 1_{A} y 1_{A}=x y 1_{A} 1_{A}=x y 1_{A}$ " suggested by the symbolic notation, in fact a syntactic manipulation without semantic control.

All three groups successfully solved question 8 , by application of the factorisation theorem to the map $\mathbb{Z} \rightarrow \mathbb{Z} / m \mathbb{Z}$. Both groups S2 and S5 relied on the diagram which proved quite effective to connect $f$ and $\bar{f}$ : since $f$ was unique by question 7 , so was $\bar{f}$. The group S4, on the contrary, did not draw such a diagram; a student of the group asked the teacher "does any map $\mathbb{Z} / n \mathbb{Z} \rightarrow \mathbb{Z} / m \mathbb{Z}$ come from a map $\mathbb{Z} \rightarrow \mathbb{Z} / m \mathbb{Z}$ ?". Another one mentioned that this result was not stated in the lecture notes and spoke about "un-quotienting". After 5 minutes of discussions within the group, the students fully realised the "universal" role played by the factorisation theorem and concluded the argument. This episode therefore confirmed the pertinence of the situation in order to illuminate the importance and rationale of proposition 1. 
5.4 Summary of empirical results and conclusions on the experiment

This experiment confirmed the importance of giving a decent amount of time to the students, to prove and apply theorems involving homomorphisms and quotients, beginning with very simple cases. The task of comparing different groups or rings of the form $\mathbb{Z} / n \mathbb{Z}$ in terms of subgroups, subrings and also quotients by means of homomorphisms allowed the students to advance their learning on homomorphisms and isomorphism theorems under the guidance of the teacher.

The epistemological idea of connecting the formalism with cognitive processes of comparison and identification brought up contrasted results. Students' relationship to abstraction and analogical thinking was itself contrasted: there were students who found it difficult to build a model of the cyclic group of order 4 inside $\mathbb{Z} / 8 \mathbb{Z}$ whereas others who more rapidly forged an abstract group concept denoted as equal isomorphic groups. Globally, identifications were related by students to equalities. This experiment showed that more didactical attention should be given to support students in the process of identifying mathematical objects under an act of abstraction, including epistemological and semiotic concerns on equivalence relations and equalities. The distinctions between equality and isomorphism, inclusion and embedding, even class and representative (in the context of isomorphisms) would need to be discussed further in order to entitle students to successfully relate the formalism of homomorphisms and the idea of comparing and identifying objects in the structuralist spirit.

From a logical point of view, $\mathfrak{S}_{1}=\mathfrak{S}_{2}$ if any property of the structure $\mathfrak{S}_{1}$ applies to $\mathfrak{S}_{2}$; the equality relates signs which are interchangeable. In the case of an isomorphism, only algebraic structural properties are preserved; moreover, the isomorphism may not be unique, and therefore the mathematicians keep different signs in order to maintain a suitable control on objects. Common mathematical practise sees the embeddings $K \hookrightarrow K[X]$ and $\mathbb{F}_{p} \hookrightarrow A$ (where $A$ denotes a ring of characteristic $p$ ) as inclusions since the homomorphisms are "canonical" (unique). This is not the case of the embedding $\mathbb{Z} / 4 \mathbb{Z} \hookrightarrow \mathbb{Z} / 8 \mathbb{Z}$. The distinction between equality and isomorphism is in fact of fundamental importance to Category Theory and has been a motivation for its development. The mathematical arguments (canonical maps, natural maps as answers to universal problems, etc.) that may be brought forward to discuss such distinctions are foreign to the students or out of reach at this stage of the learning of structuralist algebra. Therefore the intervention of the teacher is required in order to exercise mathematical control on identifications and conform to standard mathematical practise.

However, this situation proved to be quite pertinent to impart the rationale of the factorisation theorem (or mapping property of quotients) as a useful and universal formalisation. Indeed, the transition from specific rings of the type $\mathbb{Z} / n \mathbb{Z}$ to the generic ring and the question on the uniqueness of a homomorphism $\mathbb{Z} / n \mathbb{Z} \rightarrow \mathbb{Z} / m \mathbb{Z}$ led 3 groups of students (out of 5 ) to use proposition 1 , 
including the necessary condition on its hypothesis, and to reflect on the fact that any such map might be obtained by application of this proposition.

\section{Concluding remarks and perspectives}

My investigation of the teaching and learning of Abstract Algebra has been conducted under the methodology of didactical engineering and organised under the triad: epistemological analysis - textbook analysis - task design. The epistemology has highlighted the structuralist phenomenon of thematisation as a process of abstraction aiming at systematic and uniform problem solving (level 2 unification) around the central concept of homomorphism. Although algebraic concepts produce knowledge when they are tied to facts and problems, abstract concepts also become concrete when objective links are built within theories in order to form a coherent network. In this respect, emphasising thematisation in the teaching of Abstract Algebra may be seen as another way of "reducing abstraction" (Hazzan, 1999). The study of the didactic transposition of the homomorphism concept has shown contrasted teaching strategies, as the dialectic between concrete objects and abstract structures (with Category Theory as an horizon) is manifesting a tension which leads to different kinds of mathematical organisations.

With this in mind, I have chosen to anchor my teaching of homomorphisms, in the context of rings, on cognitive processes of comparison and identification involving quotient rings. The experiment has offered clues for more didactical input (in the form of meta-discourse or other means) needed to implement the potentially very fruitful epistemological idea that there is a "back-andforth play between analogy and abstraction ${ }^{13}$ " successfully in the classroom. The "theory of banquets" (a "banquet" is an invented structure simpler than group) presented in (Hausberger, 2016a) is another opportunity for discussing the concepts of homomorphism and isomorphism, yet in greater generality than the group and ring contexts. It is expected from the realisations of such activities in semi-structured interviews a deeper understanding of the students' difficulties in the conceptualisation of an abstract structure and especially thematisation.

\section{References}

Artaud, M. (1998). Introduction à l'approche écologique du didactique - L'écologie des organisations mathématiques et didactiques. In M. Bailleul, C. Comiti, J.-L. Dorier, J.-B. Lagrange, B. Parzysz, \& M.-H. Salin (Eds.), Actes de la neuvième Ecole d'été de didactique des mathématiques (p. 101-139). Caen: ARDM \& IUFM.

Artigue, M. (1991). Epistemologie et didactique. Recherches en Didactique des Mathématiques, $10(2.3)$.

\footnotetext{
${ }^{13}$ setting up analogies leads to conceive of an abstract theory and, once an abstract theory is at hand, it is used to unearth more and deeper analogies (Sinaceur, 2014, p. 98)
} 
Artigue, M. (2009). Didactical design in mathematics education. In C. Winslø w (Ed.), Nordic Research in Mathematics Education. Proceedings of NORMA08 (p. 7-16). Rotterdam: Sense Publishers.

Artin, E. (1962). Zur Problemlage der Mathematik. In S. Lang \& J. Tate (Eds.), The Collected Papers. Addison-Wesley.

Artin, M. (1991). Algebra. Englewood Cliffs, New Jersey: Prentice-Hall, Inc.

Bourbaki. (1948, reed. 1997). L'architecture des mathématiques. In F. Le Lionnais (Ed.), Les grands courants de la pensée mathématique. Paris: Hermann.

Brousseau, G. (1986). Fondements et méthodes de la didactique des mathématiques. Recherches en Didactique des Mathématiques, 7(2), 33-55.

Brousseau, G. (1997). Theory of Didactical Situations in Mathematics. Dordrecht: Kluwer.

Cavaillès, J. (2008). Oeuvres complètes de Philosophie des Sciences. Paris: Hermann.

Chevallard, Y. (1985). La transposition didactique - Du savoir savant au savoir enseigné. Grenoble : La Pensée sauvage.

Chevallard, Y., \& Bosch, M. (1999). La sensibilité de l'activité mathématique aux ostensifs. Recherches en didactique des mathématiques, 19(1), 77-124.

Corry, L. (2004). Modern Algebra and the Rise of Mathematical Structures (2nd ed.). Basel: Birkhäuser.

Dorier, J.-L., Robert, A., Robinet, J., \& Rogalski, M. (2000). The meta lever. In Dorier (Ed.), On the teaching of linear algebra (p. 151-176). Dordrecht: Kluwer Academic Publisher.

Douady, R. (1986). Jeux de cadres et dialectique outil-objet. Recherches en didactique des mathématiques, 7(2), 5-31.

Dubinsky, E. (1991). Reflective abstraction in mathematical thinking. In D. O. Tall (Ed.), Advanced Mathematical Thinking (p. 95-123). Dordrecht: Kluwer Academic Publisher.

Durand-Guerrier, V., Hausberger, T., \& Spitalas, C. (2015). Définitions et exemples : prérequis pour l'apprentissage de l'algèbre moderne. Annales de Didactique et de Sciences Cognitives, 20, 101-148.

Dyck, v. W. (1882). Gruppentheoretische Studien. Mathematische Annalen, 20(1), 1-44.

Gueudet, G. (2008). Investigating the secondary-tertiary transition. Educational Studies in Mathematics, 67(3), 237-254.

Guin, D. (1997). Algèbre Tome 1 : groupes et anneaux. Paris : Belin.

Hausberger, T. (2012). Le challenge de la pensée structuraliste dans l'apprentissage de l'algèbre abstraite : une approche épistémologique. In J.-L. Dorier \& S. Coutat (Eds.), Enseignement des mathématiques et contrat social, Enjeux et défis pour le 21e siècle, Actes du colloque EMF2012 (p. 425-434). Genève : Université de Genève.

Hausberger, T. (2016a). Abstract algebra, mathematical structuralism and semiotics. In K. Krainer \& N. Vondrová (Eds.), Proceedings of the Ninth Congress of the European Society for Research in Mathematics Education (p. 2145-2151). Prague: Faculty of Education, Charles University.

Hausberger, T. (2016b). A propos des praxéologies structuralistes en algèbre abstraite. In E. Nardi, C. Winsløw, \& T. Hausberger (Eds.), Proceedings of the 1st Congress of the International Network for Didactic Research in University Mathematics (p. 296305). Montpellier: University of Montpellier and INDRUM.

Hazzan, O. (1999). Reducing abstraction level when learning Abstract Algebra concepts. Educational Studies in Mathematics, 40, 71-90.

Lautmann, A. (2006). Les mathématiques, les idées et le réel physique. Paris : Vrin.

Leron, U., \& Dubinsky, E. (1995). An Abstract Algebra story. American Mathematical Monthly, 102(3), 227-242.

Leron, U., Hazzan, O., \& Zazkis, R. (1995). Learning group isomorphism: A crossroads of many concepts. Educational Studies in Mathematics, 29, 153-174.

Mac Lane, S. (1996). Structure in Mathematics. Philosophia Mathematica, 4(2), 174-183.

McLarty, C. (2006). Emmy Noether's "Set Theoretic" Topology: From Dedekind to the Rise of Functors. In J. Ferreiros \& J. J. Gray (Eds.), The architecture of Modern Mathematics: Essays in History and Philosophy (p. 187-208). New York: Oxford University Press, Inc. 
Nardi, E. (2000). Mathematics Undergrates' Responses to Semantic Abbreviations, Geometric Images and Multi-level Abstractions in Group Theory. Educational Studies in Mathematics, 34, 169-189.

Piaget, J. (1972). The Principles of Genetic Epistemology. London: Routledge \& Kegan Paul.

Piaget, J. (1985). The Equilibration of Cognitive Structures. Harvard University Press.

Robert, A. (1987). De quelques spécificités de l'enseignement des mathématiques dans l'enseignement post-obligatoire. In Cahier de didactique des mathématiques 47. Paris: IREM de Paris 7.

Rogalski, M. (1995). Que faire quand on veut enseigner un type de connaissances tel que la dialectique outil-objet ne semble pas marcher et qu'il n'y a apparemment pas de situation fondamentale? L'exemple de l'algèbre linéaire. In Séminaire DidaTech 169 (p. 127-162). Grenoble : Université Joseph Fourier.

Russell, B. (1903). The Principles of Mathematics. Cambridge University Press.

Scheiner, T., \& Pinto, M. (2014). Cognitive processes underlying mathematical concept instruction: the missing process of structural abstraction. In C. Nicol \& al. (Eds.), Proceedings of the Joint Meeting of PME 38 and PME-NA 36, Vol. 5 (p. 105-112). Vancouver, Canada : PME.

Simpson, A., \& Stehlíková, N. (2006). Apprehending mathematical structures: a case study of coming to understand a commutative ring. Educational Studies in Mathematics, $61,347-371$.

Sinaceur, H. (2014). Facets and Levels of Mathematical Abstraction. Philosophia Scientice, 18(1), 81-112.

Waerden, B. L. v. d. (1930). Moderne Algebra. 2 vols. Berlin: Springer.

Winsløw, C. (2008). Transformer la théorie en tâches : la transition du concret à l'abstrait en analyse réelle. In R. Rouchier \& al. (Eds.), Actes de la XIIIıème Ecole d'Eté de Didactique des Mathématiques (p. 1-12. Cédérom). Grenoble : La Pensée Sauvage.

Wussing, H. (2007). The Genesis of the Abstract Group Concept. Dover Publications. 